\section{Predicting Nitrogen Release from Parabolic-type Resin-coated Urea in Greenhouse Tomato and Cucumber Production}

\author{
Qiang Xiao ${ }^{1}$ \\ Institute of Plant Nutrition and Resources, Beijing Academy of Agriculture \\ and Forestry Sciences, Beijing 100097, China; Beijing Engineering \\ Technology Research Center for Slow/Controlled-release Fertilizer, \\ Beijing 100097, China
}

\section{XiaoHui Fan \\ Tropical Research and Education Center, Soil and Water Science Department, University of Florida, Homestead, FL 33031}

\begin{abstract}
XiaoHui Ni, LiXia Li, GuoYuan Zou, and Bing Cao ${ }^{1}$
Institute of Plant Nutrition and Resources, Beijing Academy of Agriculture and Forestry Sciences, Beijing 100097, China; Beijing Engineering Technology Research Center for Slow/Controlled-release Fertilizer, Beijing 100097, China
\end{abstract}

Additional index words. indoor incubation, pouch method, naturally varying soil temperatures, nutrient dissolved characteristics, vegetables planting

\begin{abstract}
Increasing commercial use of controlled release fertilizer (CRF) has prompted the need to predict $N$ release simply and viably in the greenhouse environment. Two CRFs were tested, i.e., P40d and P100d by incubating them for 40 or 100 days either in static water at $10,15,20,25$, and $35^{\circ} \mathrm{C}$ or in the soil of vegetable plots in a greenhouse lacking temperature controls. Cumulative nitrogen release (CNR) from a CRF was represented by a parabola curve and significantly affected by the incubation temperature. A method to calculate $N_{m}$ (the maximum $\mathbf{N}$ release percentage from CRF) was established using a firstorder kinetic equation and the method of least squares. $N_{m}$ was $90.9 \%$ to $99.9 \%$ for P40d and $72.1 \%$ to $87.1 \%$ for $\mathrm{P} 100 \mathrm{~d}$ at $10-35^{\circ} \mathrm{C}$, respectively. A relationship function between the $\mathrm{N}$ release rate and naturally fluctuating greenhouse soil temperatures was established using the activation energy of the $\mathrm{N}$ release reaction. Then a model was constructed with field temperature as the variable to predict $N$ release throughout the entire greenhouse crop production season. The value of $\psi$ representing a property of the coating material of a $C R F$ is $\approx 1.0$ for the release period of the CRF of 35-55 days and $\approx 1.2$ of 80-120 days. We validated the model using two seasons of greenhouse tomato, Solanum lycopersicum $\mathrm{L}$., and cucumber, Cucumis sativus $\mathrm{L}$., production data, and found that the error was less than $12 \%$ points. This indicated that the constructed model was sufficiently simple, practical, and accurate for use by growers, and fertilizer industry and regulatory personnel.
\end{abstract}

\footnotetext{
Received for publication 23 Jan. 2017. Accepted for publication 15 May 2017.

We would like to extend our sincere gratitude to Emeritus, Waldemar Klassen, University of Florida, for his instructive advice and useful suggestions on my thesis. We are deeply grateful of his help in the writing of this thesis. We are thankful to the national development plan focused on special projects "Developments of technology and products to preventing and controlling nitrogen and phosphorus leaching in main wheat and maize producing areas (No. 2017YFD0800604)," the National Natural Science Foundation (No. 31301847, 31301846), the Beijing Research Program of the Engineering Technology Research Center for providing the slow/controlledrelease fertilizers, and to the ecological environmental effects and evaluation of once fertilization in wheat/maize rotation system (201303103) for financial support.

${ }^{1}$ Corresponding authors. E-mail: xqiang1978@ 163.com or609284507@qq.com.
}

$\mathrm{N}$ release curve of $\mathrm{CRF}$ was divided into the sigmoid pattern, parabolic pattern, and double parabolic pattern (Yu et al., 2006). A CRF whose CNR (from a CRF) curve has a parabolic shape is called a PCRF, but if the curve is sigmoidal or " $S$ " shaped, the CRF is called a SCRF. These two CRFs have different release mechanisms and coating processes used during manufacturing. Formerly, PCRFs were used almost exclusively throughout the world. Accurate evaluations of the characteristics of $\mathrm{N}$ release from PCRFs are important for selecting a suitable release pattern of PCRF that is matched to the needs of the developing vegetable crop for optimal and efficient $\mathrm{N}$ uptake, greater yields, and reduced losses of $\mathrm{N}$ to the environment (Carson and Ozores-Hampton, 2012). Over the past 50 years, several PCRF coating technologies have been developed and marketed, and several methods to predict CNR release from these CRF technologies have been developed for regulatory purposes including 1) laboratory or growth chamber, 2) greenhouse, and 3) field (Carson and Ozores-Hampton, 2012); however, one method of CNR prediction has yet been selected for regulatory purposes in China and some other countries (Carson et al., 2014b; Fujinuma et al., 2009; Sartain et al., 2004a, 2004b).

Laboratory methods include a standard method (Dai et al., 2008; Du et al., 2006; European Committee for Standardization, 2002), and the accelerated temperaturecontrolled incubation methods (ATCIMs) (Dai et al., 2008). The earlier mentioned standard method is involved in the incubation of PCRFs, which requires the use of selected time periods, temperatures, and/or sampling methods. Compared with the standard method, ATCIMs are used with shorter incubation periods, which reduce time and labor costs. However, these two methods may be used to predict release rates in the laboratory but - by themselves - they are not able to accurately predict release rates in the field (Carson and Ozores-Hampton, 2012).

Growth chamber and greenhouse methodswhich include incubation in columns and plastic bags - may be used to test PCRF products under conditions that are fairly similar to those in a particular crop production system (Abraham and Rajasekharan Pillai, 1996; Broschat, 1996; Broschat and Moore, 2007; Sato and Morgan, 2008). The column method predicts CNR from PCRFs more accurately than the plastic bag method because ammonia volatilization and lower $\mathrm{N}$ recovery rates are associated with the bag method (Carson and OzoresHampton, 2012). During PVC-column laboratory incubation, release of PCRFs at a constant temperature and moisture content are determined by leaching the released $\mathrm{N}$ with minimal variability of incubation conditions compared with PCRFs placed in the very variable conditions of open fields (Carson et al., 2014b). Indeed, field studies are subject to diurnal temperature oscillations, variable weather patterns, and water table fluctuations (Medina, 2011). Incubation temperatures in growth chambers or greenhouses - especially greenhouses that lack precise temperature controls - tend to be more similar to soil temperatures in open fields than the testing temperatures specified by manufacturers (Carson and Ozores-Hampton, 2012).

Pot-in-pot and pouch methods are two viable field methods for evaluating PCRFs in vegetable crop production research. The pouch method-whereby water-porous pouches with PCRF prills are placed within or under vegetable beds and later recovered at predetermined times throughout the growing season-measures CNR by calculating the $\mathrm{N}$ remaining in the PCRF prills. In contrast, the pot-in-pot method-whereby the covered upper pot with a screened bottom and filled with soil from the field and mixed with PCRF prills is nested in the water-tight lower pot from which leachate is collected periodically after application 
Table 1. Collection dates and number of days since burial (in brackets) of porous pouches containing PCRFs buried $15 \mathrm{~cm}$ deep in the root-zone soil beneath the surface of black polyethylene mulch-covered beds of either a tomato or a cucumber crop during 2015 in Beijing. Samples of pouches were retrieved for analysis of $\mathrm{N}$ content on six or seven occasions throughout the crop growing season.

\begin{tabular}{|c|c|c|c|c|c|c|c|c|c|c|}
\hline \multirow[b]{2}{*}{ Fertilizer $^{z}$} & \multirow[b]{2}{*}{ Crop } & \multirow{2}{*}{$\begin{array}{c}\text { Planting } \\
\text { date }^{\mathrm{y}}\end{array}$} & \multirow{2}{*}{$\begin{array}{c}\text { Pouch } \\
\text { burial date }\end{array}$} & \multicolumn{7}{|c|}{ Pouch retrieval date } \\
\hline & & & & 1 & 2 & 3 & 4 & 5 & 6 & $7^{x}$ \\
\hline $\begin{array}{l}\text { P40d } \\
\text { P100d }\end{array}$ & $\begin{array}{l}\text { Spring } \\
\text { tomato }\end{array}$ & 22 Mar. & 24 Mar. & 31 Mar. (7 d) & 17 Apr. (24 d) & 28 Apr. $(35 \mathrm{~d})$ & 5 May (42 d) & 12 May $(49$ d) & 27 May $(64 d)^{w}$ & \\
\hline P40d & $\begin{array}{l}\text { Autumn } \\
\text { tomato and } \\
\text { cucumber }\end{array}$ & 8 Aug. & 10 Aug. & 11 Aug. (3 d) & 15 Aug. (7 d) & 4 Sept. (27 d) & 17 Sept. (40 d) & 31 Oct. $(84$ d) & 17 Nov. (101 d) & 3 Dec. $(117 \mathrm{~d})$ \\
\hline P100d & $\begin{array}{l}\text { Autumn } \\
\text { tomato and } \\
\text { cucumber }\end{array}$ & 8 Aug. & 10 Aug. & 11 Aug. (3 d) & 22 Aug. (14 d) & 4 Sept. (27 d) & 17 Sept. (40 d) & 31 Oct. $(84$ d) & 17 Nov. (101 d) & 3 Dec. $(117 \mathrm{~d})$ \\
\hline
\end{tabular}

${ }^{\mathrm{z}} \mathrm{P} 40 \mathrm{~d}$ and P100d are PCRFs. The numbers 40 and 100 indicate the number of days required for $\geq 80 \%$ of $\mathrm{N}$ in the formulation to be released.

${ }^{\mathrm{y}}$ Date when tomato and cucumber seedlings were transplanted into the soil in the greenhouse.

${ }^{\mathrm{x}}$ Number of occasions when pouches were retrieved.

${ }^{\mathrm{w}}$ Number of days that elapsed between when the pouches were buried and when they were retrieved.

of water to the upper pot-measures the instantaneous amount of $\mathrm{N}$ release leached directly from the PCRF. In any case, it is important to consider that environmental conditions in greenhouse plots and open fields are very variable, and that release rates of PCRFs depend strongly on soil temperatures. PCRF prills must meet the needs of the crop as it is influenced by fluctuating soil temperatures throughout all growing seasons and over multiple years (Fraisse et al., 2010).

Therefore, it is necessary to integrate the use of laboratory, growth chamber, and field methods into a single protocol for characterizing the performance of PCRFs under a wide range of conditions encountered in commercial vegetable production. A correlation between an ATCIM and the pouch method was developed using a two-step process in tomato, Solanum lycopersicum L. (Solanales: Solanaceae), production in Florida (Carson et al., 2014a). Japanese researchers established a model to predict CNR using field temperatures in an experiment in a rice field (Zhang et al., 2008).

Characterizing the performance of PCRFs involves a range of factors under field conditions, such as release time, temperature, moisture, placement, microbial action, and cultural practices. However, soil temperature may be considered the most influential factor influencing $\mathrm{N}$ release from PCRFs in greenhouse plots with irrigated vegetables (Carson et al., 2013; Fujita, 1989; Fujita et al., 1983).

The objective of this study was to develop and validate a model to accurately predict the $\mathrm{N}$ release characteristics of various PCRFs under the conditions of commercial vegetable crop production in nontemperatureregulated greenhouse plots. The approach to achieving this objective was to evaluate correlations between $\mathrm{N}$ release rates and soil temperatures as determined by use of a temperature-controlled incubation method and a field pouch method, to develop a predictive model based on a first-order $\mathrm{N}$ release equation that was valid under a relevant range of constant temperatures, and then to modify this equation to extend its usefulness to $\mathrm{N}$ release from PCRFs under fluctuating temperatures. We postulated that the latter step could be achieved by using the activation energy of the $\mathrm{N}$ release reaction to elucidate the relationship between the $\mathrm{N}$ release rate and the natural field temperature. The overall purpose of this effort was provide a useful predictive tool to assist growers and manufacturers to easily select PCRFs with the correct $\mathrm{N}$ release rates that match the needs of vegetable crops throughout the production process to assure efficient use of fertilizers, minimal off-site impacts, and lower economic costs.

\section{Materials and Methods}

\section{Indoor culture and field experiments}

Soil and fertilizers selected for determination of $N$ release properties. A field experiment was conducted in a greenhouse of the Beijing Academy of Agriculture and Forestry Sciences, China $\left(39^{\circ} 56^{\prime} 57^{\prime \prime} \mathrm{N}, 116^{\circ} 17^{\prime} 32^{\prime \prime} \mathrm{E}\right)$. The soil in the field was a typical cinnamon soil, i.e., a semihydromorphic soil, rich in calcium carbonate, low sandy soil, and with neutral to slightly alkaline reaction. The region has a north temperate semihumid continental monsoon climate with a mean annual temperature of $14.5^{\circ} \mathrm{C}$ and precipitation totaling $490 \mathrm{~mm}$ (Beijing Municipal Bureau of Statistics, 2015). The soil texture was loam, with a $\mathrm{pH}$ of 7.6, a bulk density of $1.2 \mathrm{~g} \cdot \mathrm{cm}^{-3}$, a field capacity of $370.3 \mathrm{~g} \cdot \mathrm{kg}^{-1}$, a nitrate-N content of $22.3 \mathrm{mg} \cdot \mathrm{kg}^{-1}$, an ammonium-N content of $4.5 \mathrm{mg} \cdot \mathrm{kg}^{-1}$, and a soil organic matter content of $11.1 \mathrm{~g} \cdot \mathrm{kg}^{-1}$. The experimental crops were the tomato, Solanum lycopersicum L. (Solanales: Solanaceae), variety 'Hard Powder 8' and the cucumber, Cucumis sativus L. (Cucurbitales: Cucurbitaceae), variety 'Beijing Green 10' (Table 1). The crops were irrigated according to typical grower practice. Details of management and cultural practices can be found in Yang et al. (2014). Two resin-coated PCRFs with different NRC periods [P40 d $(42.7 \% \mathrm{~N})$; Sanhe Xiangfengfeiye Fertilize Industry Co., Ltd., Hebei, China, and P100 d $(41.6 \% \mathrm{~N})$; Beijing Futelai Compound Fertilizers Co., Ltd., Beijing, China] were used.

CNR determination at different temperatures in the laboratory incubation. Ten grams of prills of PCRF were sealed in a nylon mesh

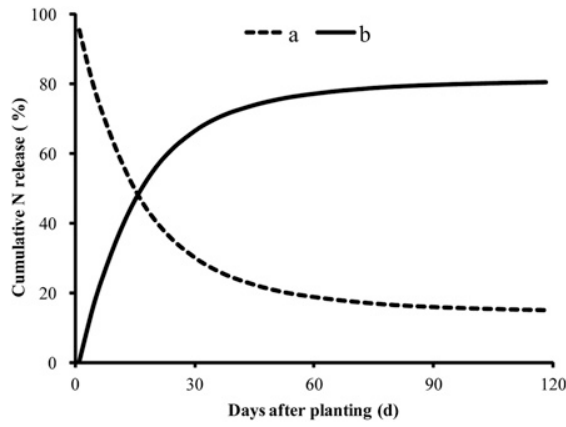

Fig. 1. N release curves pertaining to a CRF. Note: line a means a first-order reaction kinetics curve that represent the percentage of $\mathrm{N}$ that still has not been released, and line $\mathrm{b}$ means a modified first-order reaction kinetics curve that represents the cumulative percentage of $\mathrm{N}$ that has been released. Net $\mathrm{N}$ released from a CRF, as a percentage of total $\mathrm{N}$, should progressively increase with the passage of time.

bag, placed into a plastic bottle containing $250 \mathrm{~mL}$ distilled water and incubated at five different temperatures (i.e., 10, 15, 20, 25, and $35{ }^{\circ} \mathrm{C}$ ). Each treatment had four replications. The supernatant solutions were sampled at 2-d intervals in the first 20- and 5 -d intervals after $20 \mathrm{~d}$. The bottle was replaced with fresh DI water after each sampling. Total $\mathrm{N}$ in the extract solution was determined, and the total CNR from $10 \mathrm{~g}$ PCRFs since the beginning of the incubation until each sampling time was calculated (Dai et al., 2008).

CNR determination in greenhouse vegetable production. CNR from the two PCRFs in the soil of the greenhouse during tomato and cucumber production were determined in situ. Each PCRF sample-15 \pm $0.0002 \mathrm{~g}$ of the fertilizer mixed with $120 \mathrm{~g}$ of dried soil-was placed in a $15 \times 15 \mathrm{~cm}$ porous polypropylene pouch (Industrial Netting, Minneapolis, MN) with hole openings each $1.44 \mathrm{~mm}^{2}$ and $49 \%$ of the pouch's surface area consisting of holes (Carson et al., 2013). Pouches were heat-sealed and buried $15 \mathrm{~cm}$ below the soil surface, and spaced $15 \mathrm{~cm}$ between adjacent pouches along the north-south direction of the furrows. 


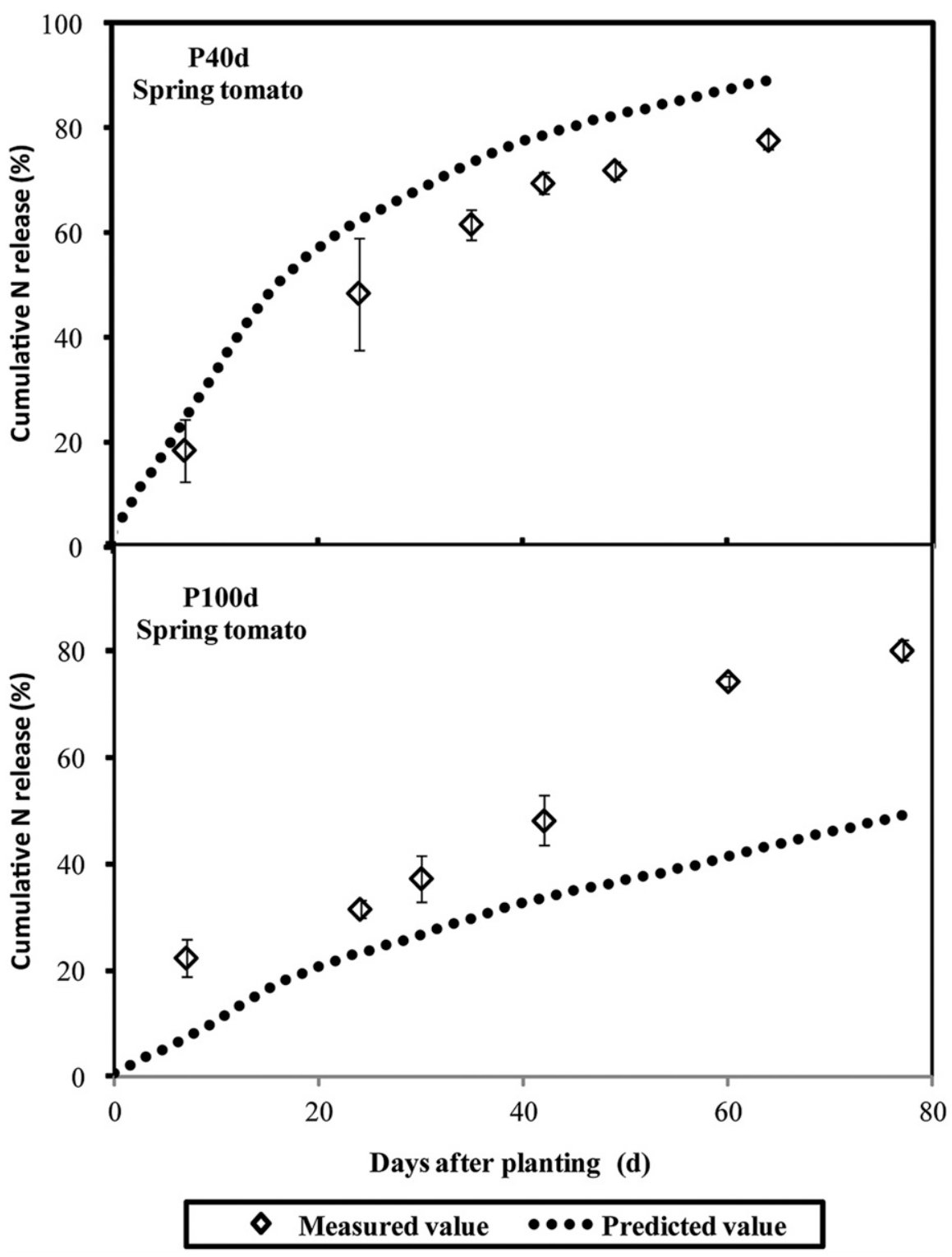

Fig. 2. Comparisons of cumulative values of $\mathrm{N}$ release from PCRFs in greenhouse plots with a spring crop of tomato between determined values and those predicted by the model of Zhang et al. (2008). Error bars represent $1 \mathrm{sD}$ from the determined means. The predicted values were substantially greater than the determined values for P40d, but for P100d, the predicted values were substantially smaller than the determined values.

The pouches were installed in each replication just on 24 Mar. and 10 Aug. 2015. Sixty pouches each of P40d and P100d were buried for an overall total of 120 . Sets of pouches were retrieved on six or seven separate occasions (Table 1) throughout the season to track the amount of nitrogen remaining in the CRF with the passage of time. The pouches were taken to the laboratory, where the fertilizer-soil mixture in each pouch was transferred onto a 2-mm sieve and washed carefully with tap water, dried in a beaker at ambient temperature and stored for analysis (Carson et al., 2012b). The samples were ground in a blender (Model 36BL23; Waring Commercial, New Hartford, CT) with $300 \mathrm{~mL}$ DI water to destroy the PCRF coating and dissolve the residual fertilizer in the pulverized remnants of the PCRF prills. The
Building the predictive model

Construction of prediction model and calculation of $N$ (CNR), $N_{m}$ (maximum release percentage), in the fertilizer. A prediction model of the $\mathrm{N}$ release from each PCRF was fitted to a first-order model (Agehara and Warncke, 2005) using the nonlinear curve-fitting PROC NLIN procedure (SAS Institute, 2011) that resulted in Eq. [1].

$$
N_{t}=N_{0} \times \exp (-k \times t)
$$

where $N_{t}$ was the cumulative percentage of the CNR from a PCRF applied at time $t\left(\mathrm{~d}^{-1}\right), N_{0}$ was the total amount - expressed as $100 \%$ of $\mathrm{N}$ in the PCRF, and $k\left(\mathrm{~d}^{-1}\right)$ was the first-order rate constant (Deans et al., 1986). The values of $N_{0}$ and $k$ were deemed significantly different $(\alpha=0.05)$ if the $95 \%$ confidence intervals did not overlap. All equations were fitted using all data points, and each point was the mean of three replications (Fan and $\mathrm{Li}, 2010$ ).

The release curve shown in Fig. 1 (line a) suggested that the $\mathrm{N}$ remaining in a PCRF decreased with the passage of time, because the slope was negative, whereas the CNR curve of $\mathrm{N}$ released from a PCRF should be the opposite with a positive slope as seen in Fig. 1 (line b). Therefore, Eq. [1] must be adjusted as shown in Eq. [2].

$$
\left(100 \%-N_{t}\right)=N_{0} \times \exp (-k \times t)
$$

Because $N_{O}$ was considered to be $100 \%$, Eq. [2] might also be converted to Eq. [3].

$$
N_{t}(\%)=N_{0} \times[1-\exp (-k \times t)]
$$

Eq. [2] may be used to tentatively describe and fit the CNR model of a PCRF under a constant temperature; however, several issues still need to be resolved.

Firstly, $N_{0}(\%)$ was intended to indicate the maximum $\mathrm{N}$ release percentage from the PCRF, which generally has been considered to have a constant value of $100 \%$, but it was not the case because the resin coat prevents the release of all $\mathrm{N}$ both in the laboratory and in the field. Accordingly, we needed to determine the maximum percentage of the $N$ in the PCRF that can be released.

However, it was necessary to first establish a new equation to determine the value of $N_{0}$. This was done as follows. The natural logarithms of both sides of Eq. [2] were taken, which resulted in Eq. [4]:

$$
\ln \left(100-N_{t}\right)=\ln N_{0}-k \times t
$$

Next, Eq. [4] was transformed into a linear regression equation, $y=a+b \times t$, in which $y=\ln \left(100-N_{t}\right), a=\ln \left(N_{0}\right)$, and $b=-k$. The linear regression equation was solved by using the least square method (Zou, 2010), then Eqs. [5]-[7] were built and values of $\mathrm{k}$, $N_{0}$, and $N_{t}$ could be obtained according to Eqs. [5]-[7]. In Eq. [6], $N_{O}$ was replaced with $N_{\text {m. }}$. Eqs. [5]-[7] were suitable for use in predicting CNR under indoor constant temperature conditions but not under the naturally fluctuating temperatures encountered either in the field or in greenhouses lacking precise temperature controls. 


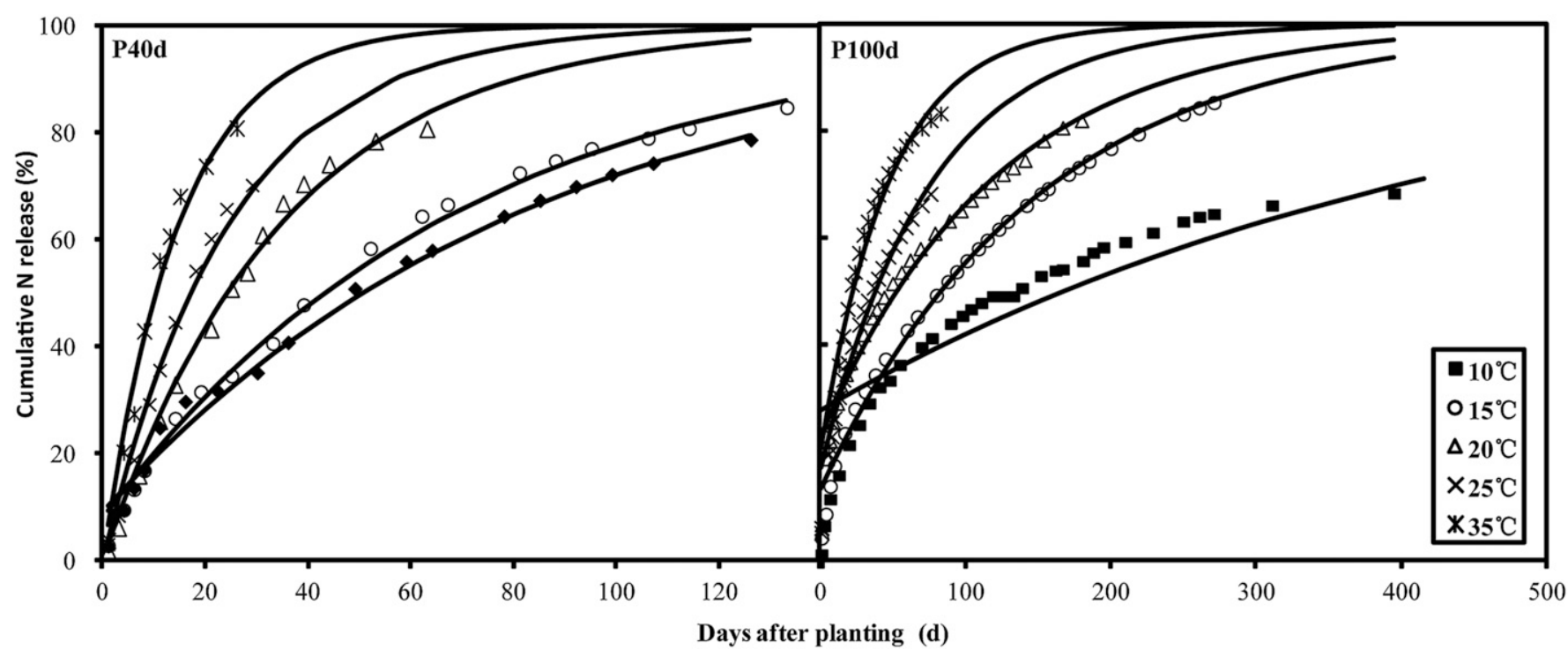

Fig. 3. Cumulative $\mathrm{N}$ release curves of $\mathrm{P} 40 \mathrm{~d}$ and $\mathrm{P} 100 \mathrm{~d}$ at $10,15,20,25$, and $35^{\circ} \mathrm{C}$, respectively. The steeper the curve, the higher the temperature and the larger the $\mathrm{N}$ release rate. The data were obtained by using a temperature-controlled incubation method with an indoor hydroponic set up.

Table 2. Regression equation-relevant parameters of cumulative $\mathrm{N}$ release from two PCRFs at $25^{\circ} \mathrm{C}$. The data were obtained by using a temperature-controlled incubation method with an indoor hydroponic set up.

\begin{tabular}{lcccrcr}
\hline CRF tested $^{\mathrm{z}}$ & $\mathrm{T}\left({ }^{\circ} \mathrm{C}\right)^{\mathrm{y}}$ & $N_{m}(\%)^{\mathrm{x}}$ & $\mathrm{K}\left(\mathrm{d}^{-1}\right)^{\mathrm{w}}$ & $t(\mathrm{~d})^{\mathrm{v}}$ & $r^{\mathrm{u}}$ & $\mathrm{Se}^{\mathrm{t}}$ \\
\hline P40d & 10 & 90.85 & 0.0118 & 130 & $0.994^{*}$ & 2.70 \\
& 15 & 92.03 & 0.0141 & 110 & $0.997^{*}$ & 2.09 \\
& 20 & 99.85 & 0.0285 & 55 & $0.998^{*}$ & 1.88 \\
& 25 & 99.96 & 0.0404 & 40 & $0.997^{*}$ & 1.93 \\
P100d & 35 & 99.79 & 0.0670 & 25 & $0.993^{*}$ & 3.08 \\
& 10 & 72.08 & 0.0022 & 450 & $0.927^{*}$ & 5.76 \\
& 15 & 87.14 & 0.0067 & 220 & $0.995^{*}$ & 2.25 \\
& 20 & 77.48 & 0.0083 & 165 & $0.983^{*}$ & 3.51 \\
& 25 & 84.00 & 0.0135 & 100 & $0.992^{*}$ & 2.65 \\
& 35 & 80.99 & 0.0212 & 68 & $0.986^{*}$ & 3.24 \\
\hline
\end{tabular}

${ }^{\mathrm{z}} \mathrm{P} 40 \mathrm{~d}$ and $\mathrm{P} 100 \mathrm{~d}$, the days required for $\geq 80 \%$ of the $\mathrm{N}$ available in the PCRF to be released from it. ${ }^{\mathrm{y}} \mathrm{T}=$ temperature.

${ }^{\mathrm{x}} N_{m}=$ the maximum percentage of the $\mathrm{N}$ available in the PCRF to be released from it.

${ }^{\mathrm{w}} \mathrm{K}=$ constant in the first-order kinetic equation for nitrogen release rate from a PCRF.

${ }^{\mathrm{v}} t=$ the days required for $\geq 80 \%$ of the releasable $\mathrm{N}$ in the PCRF to be released.

u $r$ obtained by using a regression equation. *Significant at $P \leq 0.05$.

${ }^{t}$ Root mean square error for determined and regression values.

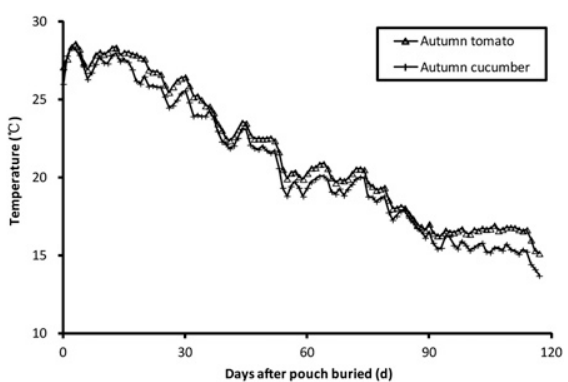

Fig. 4. Soil temperatures in the greenhouse during the autumn growth period of tomato and cucumber. Soil temperature had an effect on $\mathrm{N}$ release from the PCRFs in buried pouches. As the vegetable growth and maturation season progressed, the soil temperature gradually declined, and the differences of the soil temperatures in the tomato and cucumber plots were not significant. greenhouse with fluctuating temperatures. There were several algorithms for the introduction of field soil temperatures into a predictive model, but the days of temperature switch (DTS) algorithm (Zhang et al., 2008) was finally selected through a process of screening and comparison and was done using Eq. [8]. And then, the release time, $t_{s}$-number of days at constant indoor $25^{\circ} \mathrm{C}$ obtained from Eq. [8]was entered into Eq. [7], in which the values of the other parameters were obtained from a $25^{\circ} \mathrm{C}$ static water incubation experiment. In this way, we obtained the instantaneous amount of CNR in greenhouse plots in which the soil temperature fluctuated.

$$
t_{s}=\exp \left[\frac{E a\left(T_{i} T_{s}\right)}{R T_{i} T_{s}}\right]
$$

where $t_{s}(\mathrm{~d})$ was the days of cumulative CNR at standard temperature, $E a\left(\mathrm{~J} \cdot \mathrm{mol}^{-1}\right)$ was the activation energy of the reaction, $R$ $\left(8.314 \mathrm{~J} \cdot \mathrm{mol}^{-1}\right)$ was the gas constant, and $T_{i}$ was the absolute temperature $(\mathrm{K})$ under any natural condition, $T_{s}$ was for the standard absolute temperature (298 K). The calculation of $E a$ was very important and was based on the Arrhenius formula (Eq. [9], Song, 1988). Ea and the pre-exponential factor $(A)$ can be calculated by the linear least square method.

$$
E_{a}=(\ln A-\ln k) \times R \times T
$$

where $k\left(\mathrm{~d}^{-1}\right)$ was the release rate constant at a certain temperature, $A$ was the frequency factor, $E a\left(\mathrm{~J} \cdot \mathrm{mol}^{-1}\right)$ was the activation energy of the reaction, $R\left(8.314 \mathrm{~J} \cdot \mathrm{mol}^{-1}\right)$ was the gas constant, and $T(\mathrm{~K})$ was the absolute temperature. Parameter $k$ can be measured at several known temperatures, $E a$ and $A$ can be calculated by either of the two-point method, the linear plot method or the linear least squares method. Compared with the other two methods, the linear least squares method 
can provide the most accurate estimation of the activation energy although the calculation was more complex. However, we found that the prediction error was still large if the DTS was applied directly to Eq. [7] (Fig. 2).

On this base, experiments of spring tomato and cucumber crops grown in greenhouse plots were involved to build the parameter, $\psi$, to modify Eq. [7] and Eq. [10] was the result at last.

$$
N_{t}=N_{m} \times\left[1-\exp \left(-k \times \sum_{s=1}^{n} t_{s}\right)\right] \times \psi
$$

The factor, $\psi$, represented a property of the coating material of a PCRF-whose value ranges between 1.0 and 1.2, and $\psi$ was an independent variable in the modified prediction model. According to our many fitting results and the release laws of CRF in general, the value of the $\psi$ factor was $\approx 1.0$ when the designated CNR period to release $N_{m}$ was $35-55 \mathrm{~d}$. However, the value of $\psi$ was $\approx 1.2$ when the designated CNR period to release $N_{m}$ was $80-120$ d.

Having explained how we derived the prediction model and the methods of calculating its parameters, we would give examples to illustrate the construction process of the prediction model, and verify and revise the prediction model.

\section{Results}

Calculation of the CNR from PCRF and its related parameters. CNR curves at five temperatures (Fig. 3) were obtained by using an indoor hydroponic set up. The CNR curves at the five temperatures had the characteristics of a parabolic release curve. $N_{m}$ (maximum $\mathrm{N}$ release amount) and $k$ (N release rate) were calculated by Eqs. [5]-[7] (Table 2). Results showed that the relative coefficient, $r$, values were greater than 0.993 and there were strongly significant correlations between CNR rates and temperatures. The higher the temperature, the steeper the curve. The $N_{m}$ values of the P40d and P100d PCRFs were $90.9 \%$ to $99.9 \%$ and $72.1 \%$ to $87.1 \%$ at temperatures in the range $10-35{ }^{\circ} \mathrm{C}$, respectively. These results showed that the values obtained from the regression equations fairly closely matched the determined values.

The linear least squares method and Eq. [9] were applied to calculate the $E a$ values of P40d and P100d, which were 57,421 J.mol ${ }^{-1}$ and $65,903 \mathrm{~J} \cdot \mathrm{mol}^{-1}$, respectively. Each of these $E a$ values was a constant in the range of $10-35^{\circ} \mathrm{C}$.

Characteristics of CNR, modification of parameters, and model verification in greenhouse soil. To illustrate the predictive accuracy of the model, additional greenhouse plot experiments were carried out to determine the CNR characteristics of the two PCRFs, i.e., P40d and P100d, using the pouch-field method in autumn tomato and cucumber plots in the greenhouse.
The average soil temperatures at the same depth as the buried pouches with the two PCRFs decreased gradually with the passage of time from 8 Aug. to 3 Dec., and the average soil temperature was lower than $25^{\circ} \mathrm{C}$ from 30 to $120 \mathrm{~d}$ (Fig. 4). The rate of CNR trended progressively lower much like the soil temperature. However, the release time required for the same amount of CNR was different between the indoor and greenhouse experiments. Furthermore, the characteristic of their CNR curves were substantially different (Figs. 5 and 6), and these differences were mainly caused by the variable greenhouse soil temperatures. The days required for the P40d and P100d formulations to release $80 \%$ of their releasable $\mathrm{N}$ contents in the greenhouse soil with autumn tomato were 55 and $150 \mathrm{~d}$, respectively. These periods were 15 and $50 \mathrm{~d}$ longer, respectively, than in the indoor experiment with a constant temperature of $25^{\circ} \mathrm{C}$. Similar results were obtained in the experiment with the plot of autumn cucumber.

This study showed that the variable field temperatures led to substantial differences in the CNR between incubation in an indoor constant temperature water bath and the greenhouse soil with fluctuating temperatures used for vegetable production. The longer the NRC period - of the PCRF under the conditions of a constant temperature - the greater the difference between the CNR determined under fluctuating incubation temperatures.

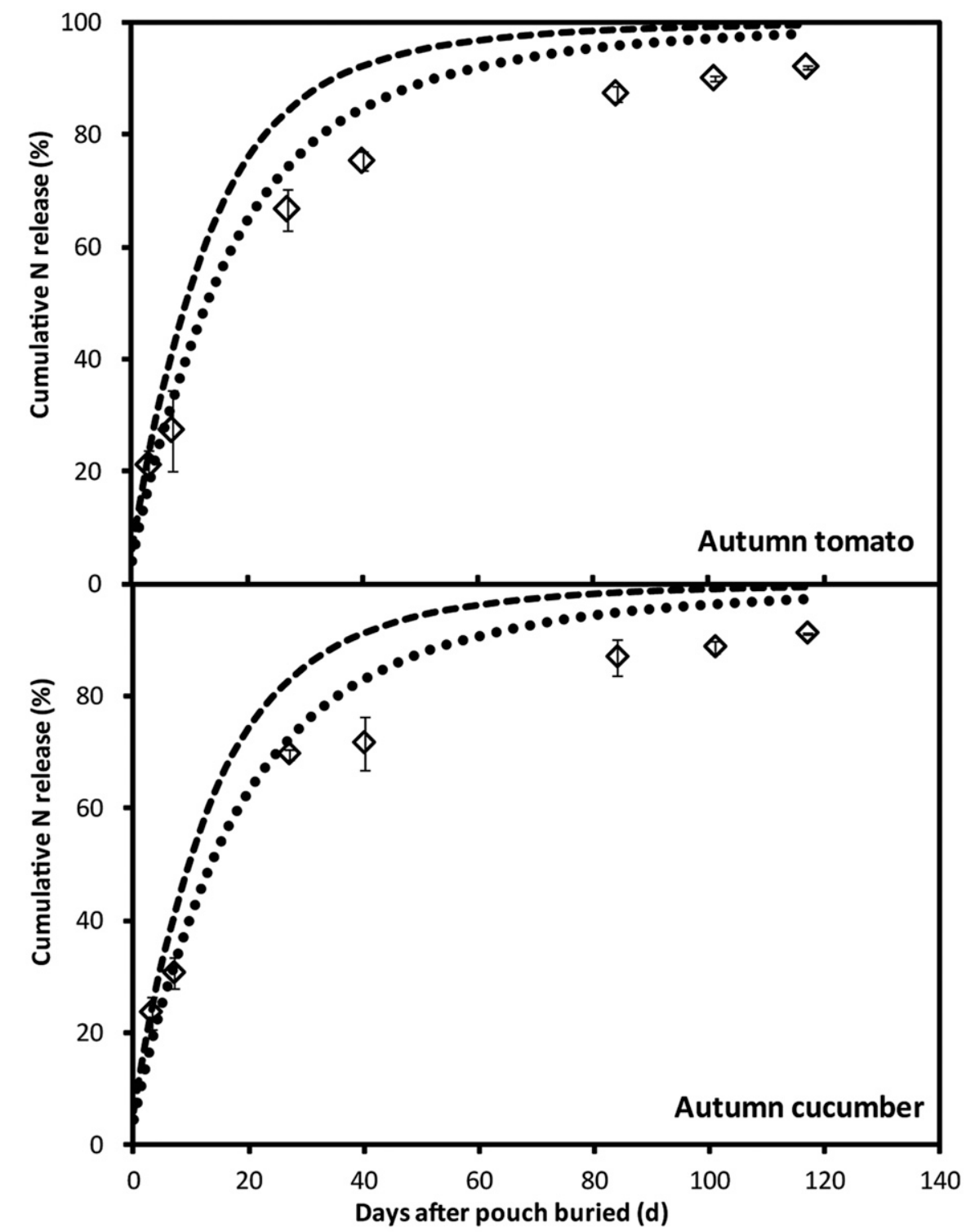

๑ Measured value $\bullet \bullet \bullet \bullet$ Predicted value1 — - Predicted value2

Fig. 5. Comparing the prediction results of Eq. [10] and Zhang et al. (2008)'s model between predicted and determined values of cumulative $\mathrm{N}$ release from $\mathrm{P} 40 \mathrm{~d}$ in the soils of greenhouse plots of autumn tomato and cucumber growth. Error bars represent $1 \mathrm{sD}$ from the determined means. The differences between determined and predicted value 1 of Eq. [10] were small and the error was less than $12 \%$ points of $\mathrm{N}$ released in the soil in autumn tomato and cucumber plots, but the errors between determined and predicted value 2 of Zhang et al. (2008)'s model were greater than that of Eq. [10]. 


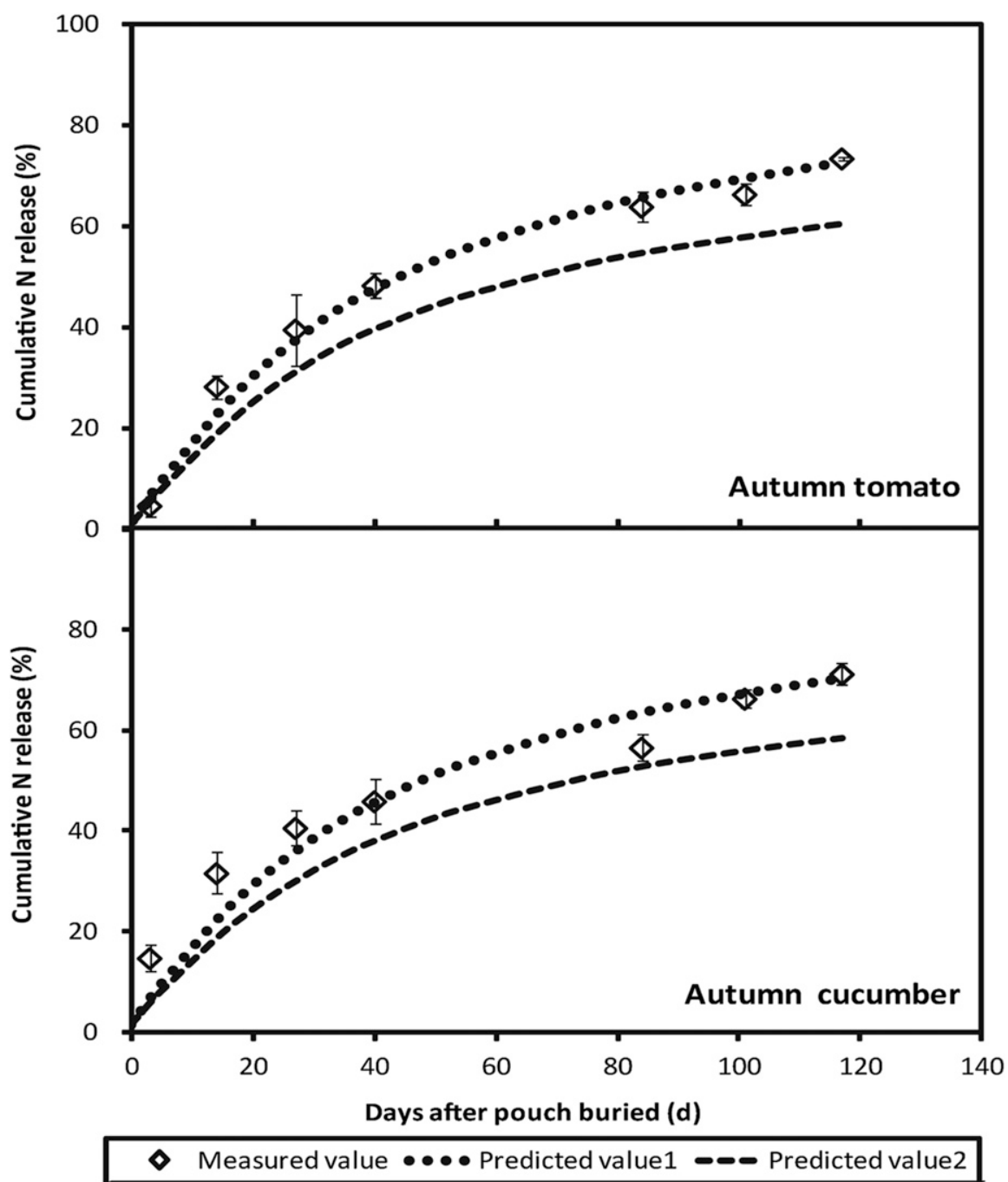

Fig. 6. Comparing the prediction results of Eq. [10] and Zhang et al. (2008)'s model between predicted and determined values of cumulative $\mathrm{N}$ release from P100d in the greenhouse field with autumn tomato and cucumber growth. Error bars represent $1 \mathrm{SD}$ from the determined means. The difference between determined and predicted value 1 of Eq. [10] were small and the error was considerably less than $12 \%$ points in autumn tomato and cucumber plots, but the errors between determined and predicted value 2 of Zhang et al. (2008)'s model were greater than that of Eq. [10].

The experiments showed that $\mathrm{P} 40 \mathrm{~d}$ and P100d released $70 \%$ to $87 \%$ and $30 \%$ to $44 \%$ of the releasable $\mathrm{N}$ by $30-46 \mathrm{~d}$, respectively, after the tomatoes had been transplanted. Similar results were obtained in the experiment involving autumn cucumber. This showed that the law of CNR from a given PCRF is valid at each soil temperature regardless of the crop cultivar or species and the uptake of nitrogen. The CNR in greenhouse plots that were predicted by the model may assist growers in selecting a specific CRF with a suitable release rate and duration to meet the needs of tomato and cucumber crops (Carson et al., 2014c).

On the basis of the above results and analysis, we can better estimate the parameter values of the model to predict the CNR. Daily records of soil temperature (Fig. 4) in the greenhouse were input into Eq. [8], followed by $\mathrm{N}$ release days $\left(t_{s}\right)$ and the cumulative release days $\left(\sum_{s=1}^{n} t_{s}\right)$ at a $25^{\circ} \mathrm{C}$ constant indoor temperature. The $\left(\sum_{s=1}^{n} t_{s}\right)$ and other values of parameters of $k, N_{m}$ at a $25^{\circ} \mathrm{C}$ constant indoor temperature (Table 2) and the appropriate value of $\psi$ selected- $-\psi$ values corresponding to $\mathrm{P} 40 \mathrm{~d}$ and $\mathrm{P} 100 \mathrm{~d}$ were 1.0 and 1.2, respectively, according to Fig. 3, which were entered into Eq. [10] during an autumn tomato and cucumber crops in the greenhouse. By comparing the predicted value 1 and determined value (Figs. 5 and 6), the errors between them were found to be less than $12 \%$ points. However, when the model of Zhang et al. (2008) was applied to predict, the errors between predicted value 2 and determined value were found to be more than $12 \%$ points (Figs. 5 and 6). Three problems in this model were found that might influence the accuracy of prediction: 1) the method of constructing this model was flawed, 2) the method might not be suitable for the prediction of CNR for all PCRFs in China because of certain differences in characteristics of the various kinds of CRFs, such as coating materials, and 3) it was not clear whether this model could predict the CNR in greenhouse vegetable production in China because of differences in the properties of various soils used for vegetable production and differences in cultivation practices among crop species and among various growers. Therefore, our application the model of Zhang et al. (2008) had been modified. The critical problem in the calculation process involving DTS (Zhang et al., 2008) was that the value of the product, $k \times t$, required by the same amount of CNR for different temperatures was thought to be constant so that $t_{1} \times k_{1}=t_{2} \times k_{2}=\ldots t_{n} \times k_{n}$. However, this might be not true, and was found that the $k \times t$ product could have a wide range of values. Thus, the assumption that $t_{1} \times k_{1} \approx t_{2} \times k_{2} \approx \ldots \approx t_{n} \times k_{n}$, might produce quite erroneous predictions (Fig. 2). To solve this, the factor, $\psi$, had been built to solve the problem. This indicates that our model can accurately predict the CNR of a PCRF under the fluctuating greenhouse soil temperature conditions. This demonstrates that more accurate CNR curves of PCRFs can be provided to producers and growers, and the predictive value of these curves can provide them with valuable guidance.

In addition, our prediction method was currently only available for thermoplastic material-coated fertilizers and might be not for nonthermoplastic membrane materials which were not sensitive to temperature. Indeed, the CNR rates of PCRFs with nonthermoplastic membrane materials may not be related to temperature. In contrast, thermoplastic film materials were affected strongly and swiftly by temperature changes so that the CNR rate may be affected greatly. Although some researchers have used ATCIMs to predict the amount of CNR in laboratory studies, more research would be needed. It was found that some of the coating membranes were broken or agglomerated when the temperature rose sufficiently high, which caused abnormal CNR and affected the predicted NRC periods of the affected PCRFs. In fact, many studies showed that ATCIMs were unsatisfactory for predicting CRFs with very long NRC periods (Dai et al., 2008; Medina et al., 2009; Sartain et al., $2004 a, 2004 b$ ). Based on all of the above considerations, parameters such as $K$ and $N_{t}$ in the $10-35^{\circ} \mathrm{C}$ range were examined in this study. The variability of these parameters in the $10-35{ }^{\circ} \mathrm{C}$ range was less than that at higher temperatures, and this was also the case with the reactive kinetic parameters. Moreover, the CNR rate remained relatively stable. Furthermore, the annual range of soil temperatures in the greenhouse plots was 13-35 ${ }^{\circ} \mathrm{C}$ without the occurrences of higher temperatures (Fig. 4), which was consistent with the temperatures used in our experiments. Therefore, the values of released $\mathrm{N}$ predicted by the model were closer to the values determined in the field than those reported from indoor experiments (Carson and OzoresHampton, 2012; Carson et al., 2014b; Medina et al., 2009; Sartain et al., 2004a, 2004b).

Our main purpose in building the predictive model was to improve the accuracy of 
predictions by means of a fairly simple and practical approach. In this study, the limiting value of error in the accuracy of prediction of $\mathrm{N}$ release was less than $12 \%$ points; although this value was greater than has been achieved in the laboratory with some purely rational models, it was smaller than had been achieved for greenhouse fields. Additional improvements in our model should be possible. Thus, the factors of different soil types also may need to be considered. Our model was suitable for application in cinnamon soil currently. Although we did not study the prediction in other soil types, we thought the model might be suitable for use in loam or clay as long as sufficient moisture. For sandy soil, the applicability of our model need to be further researched, because of its simple structure and relative difficulties of water moving.

\section{Conclusions}

$\mathrm{N}$ release data from PCRFs-obtained through a combination of incubation experiments conducted at a constant temperature indoors and in the soil with naturally fluctuating temperatures in a nontemperature regulated greenhouse - were critically important in developing a model to predict rates and durations of CNR of various PCRFs. The $N_{m}$ value of each PCRF could be obtained based on a first-order kinetic equation transformation and the least square method the data obtained in the above indoor incubation experiments at five different temperatures. This allowed the formulation of an equation to calculate the percentages of $\mathrm{N}$ in PCRFs that have the potential to be mineralized at a constant temperature. This equation was modified to allow estimates of the release of $\mathrm{N}$ from PCRFs under fluctuating temperatures by using the activation energy of the $\mathrm{N}$ release reaction to elucidate the relationship between the N release rates of each PCRF and the naturally occurring field temperatures. However, the resulting equation allowed the reliable prediction of CNR only for periods of 35-55 d. To make the equation fit the observed CNR data for PCRF with NRC periods $80-120 \mathrm{~d}$, a factor, $\psi$ - which represents a property of the coating material of the PCRF, and whose value was 1.2 for PCRFs with long NRC periods - was introduced into the prediction equation. This resulted in a simple, viable model that allows prediction of CNR rates of PCRFs with an error of less than $12 \%$ points. Future research should focus on reducing this inherent error in predictions.

\section{Literature Cited}

Abraham, J. and V.N. Rajasekharan Pillai. 1996. Membrane-encapsulated controlled-release urea fertilizers based on acrylamide copolymers J. Appl. Polym. Sci. 60:2347-2351.

Agehara, S. and D.D. Warncke. 2005. Soil moisture and temperature effects on nitrogen release from organic nitrogen sources. Soil Sci. Soc. Amer. J. 69:1844-1855.

Beijing Municipal Bureau of Statistics. 2015. Beijing statistical yearbook. China statistics press, Beijing, China. 20-50 (In Chinese).

Broschat, T.K. 1996. Release rates of soluble and controlled-release potassium fertilizers. HortTechnology 6:128-131.

Broschat, T.K. and K.K. Moore. 2007. Release rates of ammonium-nitrogen, nitrate-nitrogen, phosphorus, potassium, magnesium, iron, and manganese from seven controlled-release fertilizers. Commun. Soil Sci. Plant Anal. 38:843850.

Carson, L.C. and M. Ozores-Hampton. 2012. Methods for determining nitrogen release from controlled-release fertilizers used for vegetable production. HortTechnology 22:20-24.

Carson, L.C., M. Ozores-Hampton, and K.T. Morgan. 2013. Nitrogen release from controlled- release fertilizers in seepage-irrigated tomato production in south Florida. Proc. Annu. Meet. Fla. State Hort. Soc. 126:131-135.

Carson, L.C., M. Ozores-Hampton, K.T. Morgan, and J.B. Sartain. 2014a. Nitrogen release properties of controlled-release fertilizers during tomato production. HortScience 49:1568-1574.

Carson, L.C., M. Ozores-Hampton, K.T. Morgan, and J.B. Sartain. 2014b. Prediction of controlledrelease fertilizer nitrogen release using the pouch field and accelerated temperature-controlled incubation methods in sand soils. HortScience 49:1575-1581.

Carson, L.C., M. Ozores-Hampton, and J.B. Sartain. 2012b. Controlled-release fertilizer drying methods effect on nitrogen recovery analysis. HortScience 47:S320.

Dai, J.J., X.L. Fan, J.G. Yu, F. Liu, and Q. Zhang. 2008. Study on a rapid method to predict longevity of controlled release fertilizer coated by water soluble resin. Agr. Sci. China 7:11271132. (In Chinese).

Deans, J., J. Molina, and C. Clapp. 1986. Models for predicting potentially mineralizable nitrogen and decomposition rate constants. Soil Sci. Soc. Amer. J. 50:323-326.

Du, C.W., J.M. Zou, and A. Shaviv. 2006. Release characteristics of nutrients from polymercoated compound controlled release fertilizers. J. Polymers Environ. 14:223-230.

European Committee for Standardization. 2002. Slow-release fertilizers: Determination of the nutrients-method for coated fertilizers. EN 13266:2001. European Committee for Standardization, Brussels, Belgium.

Fan, X.H. and Y.C. Li. 2010. Nitrogen release from slow-release fertilizers as affected by soil type and temperature. Soil Sci. Soc. Amer. J. 74:16351641.

Fraisse, C.W., Z. Hu, and E.H. Simonne. 2010. Effect of El Nino-southern oscillation on the number of leaching rain events in Florida and implications on nutrient management for tomato. HortTechnology 20:120-132.

Fujinuma, R., N.J. Balster, and J.M. Norman. 2009. An improved model of nitrogen release for surface applied controlled-release fertilizer. Soil Sci. Soc. Amer. J. 73(6):2043-2050.

Fujita, T. 1989. Invention and development of polyolefin-coated urea. Ph.D.dissertation. Tohoku Univ., Sendai, Japan.

Fujita, T., C. Takahashi, S. Yoshida, and H. Himizu. 1983. Coated granular fertilizer capable of controlling the effects of temperature on dissolution out rate. U.S. Patent 4369055. 18 Jan. 1983.

Medina, L.C., J.B. Sartain, and T.A. Obreza. 2009 Estimation of release properties of slow-release fertilizer materials. HortTechnology 19:13-15.

Medina, C. 2011. Method development to characterize nutrient release patterns of enhanced efficiency fertilizers. Univ. Florida, Gainesville, FL, PhD Diss.

Sartain, J.B., W.L. Hall, R.C. Littell, and E.W. Hopwood. 2004a. Development of methodologies for characterization of slow-release fertilizers. Proc. Soil Crop Sci. Soc. Fla. 63:72-75.

Sartain, J.B., W.L. Hall, R.C. Littell, and E.W. Hopwood. 2004b. New tools for the analysis and characterization of slow-release fertilizers, p. 180-195. In: W.L. Hall and W.P. Robarge (eds.). Environmental impact of fertilizer on soil and water. American Chemical Society, Washington, DC.

SAS Institute. 2011. SAS/STAT 9.3 User's Guide. SAS Inst., Cary, NC.

Sato, S. and K.T. Morgan. 2008. Nitrogen recovery and transformation from a surface or sub-surface application of controlled-release fertilizer on a sandy soil. J. Plant Nutr. 31:2214-2231.

Song, Y.D. 1988. Arrhenius formula and activation energy. J. Shanxi Agr. Univ. 8(2):230-235.

Yang, J.G., X.H. Ni, B. Cao, Q. Xiao, G.Y. Zou, and B.C. Liu. 2014. Effect of special controlled-release fertilizer application on nitrogen and potassium uptakes of tomato and their residue in soil with drip-irrigation in the green-house. J. Plant Nutr. Fert. 20(5):1294 1302. (In Chinese).

Yu, J.G., X.L. Fan, N. Li, and F. Liu. 2006. Application of the richards equation to describe nitrogen release characteristics from controlled release fertilizer (CRF). Zhongguo Nong Ye Ke Xue 39(9):1853-1858. (In Chinese).

Zhang, Y.L., Y.L. Zhang, X.L. Dang, W.S. Yin, and L.P. Zhu. 2008. Prediction on the dissolving rate of nitrogen from coated urea by days of temperature switch. Chin. J. Soil Sci. 39(3):582585. (In Chinese).

Zou, L. Q. 2010. Principle of least square method and its simple application. Science \& Technology Information 23:282-283. (In Chinese). 